\title{
Airborne megawatt class free-electron laser for defense and security
}

\author{
Roy Whitney*, David Douglas, George Neil \\ Jefferson Lab, 12000 Jefferson Avenue, Newport News, VA USA 23606
}

\begin{abstract}
An airborne megawatt (MW) average power Free-Electron Laser (FEL) is now a possibility. In the process of shrinking the FEL parameters to fit on ship, a surprisingly lightweight and compact design has been achieved. There are multiple motivations for using a FEL for a high-power airborne system for Defense and Security:

- Diverse mission requirements can be met by a single system.

- The MW of light can be made available with any time structure for time periods from microseconds to hours, i.e. there is a nearly unlimited magazine.

- The wavelength of the light can be chosen to be from the far infrared (IR) to the near ultraviolet (UV) thereby best meeting mission requirements.

- The FEL light can be modulated for detecting the same pattern in the small fraction of light reflected from the target resulting in greatly enhanced targeting control.

The entire MW class FEL including all of its subsystems can be carried by large commercial size airplanes or on an airship. Adequate electrical power can be generated on the plane or airship to run the FEL as long as the plane or airship has fuel to fly. The light from the FEL will work well with relay mirror systems. The required R\&D to achieve the MW level is well understood. The coupling of the capabilities of an airborne FEL to diverse mission requirements provides unique opportunities.
\end{abstract}

Keywords: Free-electron laser, FEL, laser, airborne, megawatt, airship, relay mirror

\section{INTRODUCTION}

Airborne MW class FELs can be scaled up and down in power levels to be considered for multiple mission areas including theater missile defense, forward base defense, U.S. perimeter defense. This airborne FEL based capability is largely the result of the Navy's investment at Jefferson Lab in FEL development for shipboard missile defence. A lightweight and compact shipboard based design has been achieved that can be further compacted for airborne and airborne transported missions.

\subsection{FEL Motivations}

FELs are very attractive for a variety of missions because their optical beams can reach full power in a few seconds, the wavelengths of light can be chosen that best meet mission needs, and they have unlimited magazines. The challenge has been to demonstrate that they can achieve high power levels and that they can be compacted enough to be quickly placed in desired locations. The roadmap to high power levels is being actively worked. This paper focuses on how to achieve compact and light weight total system designs and the resulting mission opportunities.

\subsection{Jefferson Lab FEL}

The largely Navy funded Jefferson Lab FEL ${ }^{1,2,3,4}$ (Fig. 1) is a spinoff of the technologies developed to accomplish the U.S. Department of Energy Office of Science's nuclear physics mission at the Lab: performing fundamental research into the nature of matter at the quark and gluon level ${ }^{5}$. To achieve this mission, the Lab deployed the largest installation of superconducting radiofrequency (SRF) in the world for the Continuous Electron Beam Accelerator Facility. Significant advances in SRF and Energy Recovering Linac ${ }^{6,7}$ (ERL) technologies enabled the development of the world's highestaverage-power FEL. Recently (July 2004) the Lab's FEL achieved 10,000 watts in the IR range at 6 microns. During early 2005 the FEL is being operated at 3 and 1 microns with the goal of 10,000 watts output. Mid 2005 installation of a second wiggler funded by the Air Force will be completed and over 1,000 watts of extracted beam in the UV range is planned. In

*whitney@jlab.org; phone 757.269.7536; www.jlab.org 
addition, by mid 2005 Army funding will provide 100 watts in the terahertz range for experiments, ${ }^{8,9}, 10$ a power level that is many orders of magnitude higher then any other source in the world.

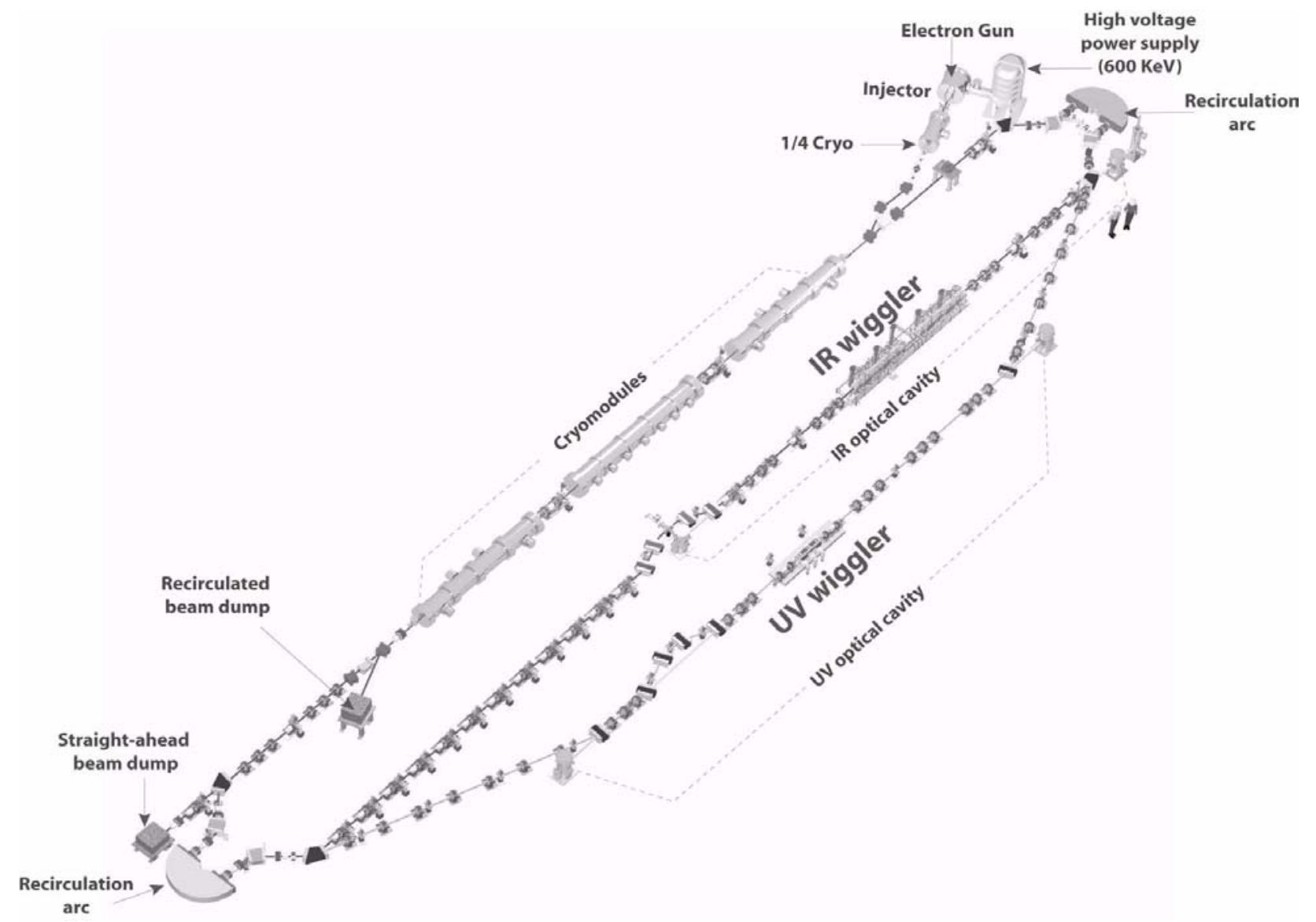

Figure 1: Jefferson Lab FEL. The IR beam achieves 10,000 watts and the UV beam will achieve 1,000 watts. The 100 watts of terahertz can be sourced from several locations.

\subsection{Compact FEL}

While the Jefferson Lab FEL has been designed as a research device with an expanded layout for design studies, a Navy goal is to achieve a compact device that can be placed on a variety of ships. Using advanced accelerator physics optics concepts $^{11}$, this goal has been met at the design level as shown in Figure 2. Continuing advances in SRF and ERL technologies have resulted in a compact FEL with only an 18 meter long optical cavity. The SRF advances mean that only a single cryomodule will be required as compared with the three in the present FEL. The ERL advances have been the result of understandings achieved for beam properties from the present FEL and theoretical achievements in the area of high power electron beams. What is also impressive is that this compact FEL is surprisingly lightweight as will be discussed in the next section. This combination of the small size and lightweight creates the opportunity for placing FELs in the airborne environment.

The challenge becomes achieving compact and lightweight auxiliary systems for electrical power, cooling and the superfluid liquid He refrigeration system required for the SRF cavities. 


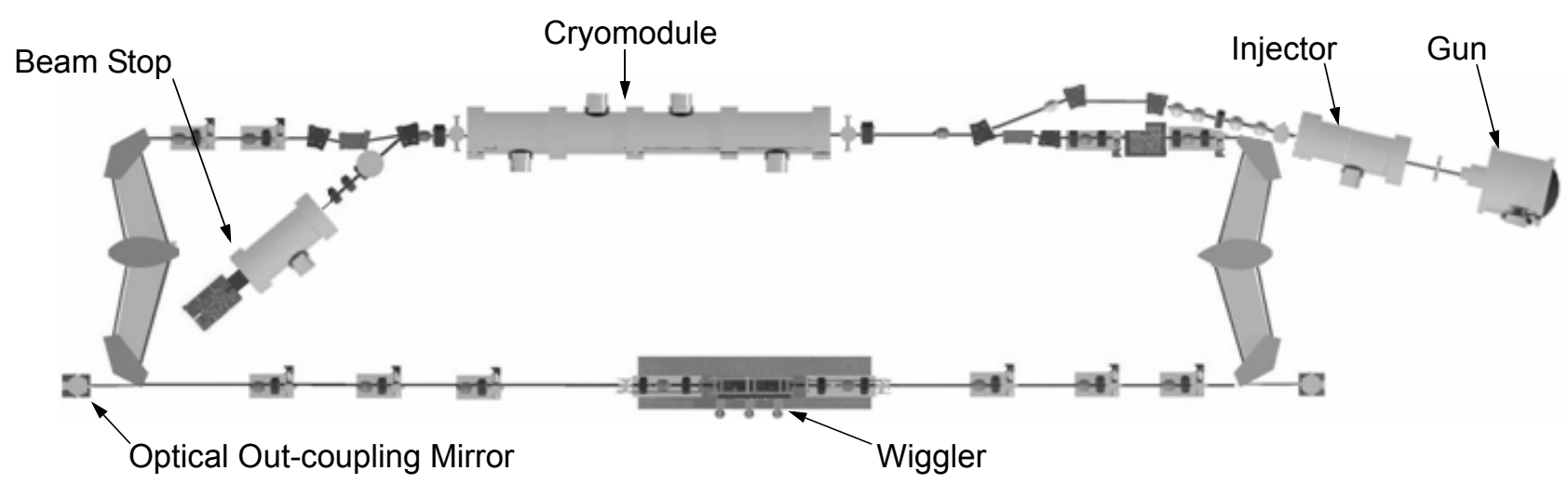

Figure 2: Advanced accelerator physics optics concepts were used to achieved the design of a compact FEL. ${ }^{11}$ The total length of the optical cavity is 18 meters. The system design is for MW and higher capabilities in the extracted optical beam. The injector, cryomodule and beam stop all have SRF cavities. The electron beam from the photocathode is accelerated to $0.5 \mathrm{MeV}$ in the DC gun and then to $7.5 \mathrm{MeV}$ in injector. The cryomodule accelerates the beam to $80-160 \mathrm{MeV}$ depending upon the wavelength of light desired. The high-power optical beam is created in the wiggler. Approximately $99 \%$ of the electron beam's energy remains after the wiggler and using ERL principles, it is recovered by passing the beam with reverse phase back through the cryomodule. An SRF cavity in the beam stop removes most of the energy that was supplied by the injector. This RF power is returned to the Injector. A small amount of energy is absorbed at the end of the beam stop.

\section{AIRBORNE FEL SYSTEM}

A key to successfully operating a MW class FEL on an airplane or airship is demonstrating that mission requirements can be met for size, weight, power, cooling, etc. A Boeing 747 will be used as an example airplane because of its wide use and ease in meeting the requirements. A variety of other airplanes could also be used. For the airships discussed later in the paper, designs that could easily lift an FEL have been developed.

\subsection{MW FEL on Boeing 747}

Figure 3 shows the compact FEL in a Boeing 747. All of the FEL accelerator and optical components easily fit within the cargo area. The gas compressors/expanders external to the cold box and the electric generator have been placed under the wings where they can be powered by auxiliary jet engines. An option may be to place all of these subsystems in a single under-wing pod driven by a single auxiliary jet engine. The high power laser beam is transported to the directional mirror in nose of the airplane.

\subsection{Altitude, Range, Time on Station, and Weight Considerations}

To meet mission requirements, the airplane with the FEL needs to operate at 40,000 feet and have a maximum range and time on station. A Boeing 747 cargo plane can carry $248,300 \mathrm{lbs}^{12}$. However, carrying this much weight would put limitations on the time on station at 40,000 feet. Consequently, a goal of 180,000 lbs is being used as the maximum weight for the FEL and all of its subsystems. This will also allow capacity for other systems of interest. 


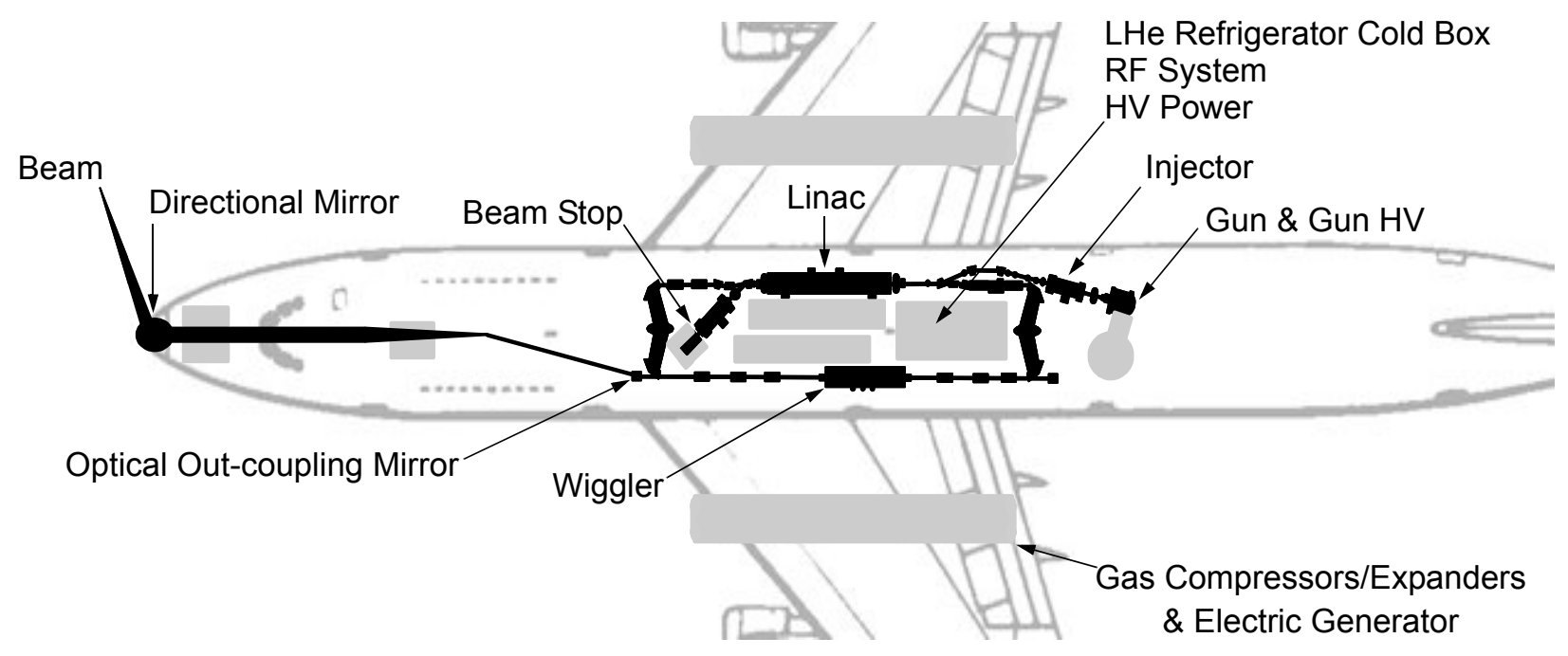

Figure 3: Compact FEL on a 747. The cargo bay easily carries the FEL itself and all of the auxiliary equipment that operates in room temperature and pressure environments. The gas compressors/expanders external to the cold box and electric generator systems are powered by auxiliary jet engines and placed under the wings.

\subsection{FEL Subsystems and Weights}

Table 1 provides a listing of the FEL subsystems and their weights. Following are important points in the design with respect to weights:

- The weights for the mechanical support for subsystem outside of the main cabin are included with the weight of the components.

- The rotating power is being taken directly off a jet engine for the high pressure He screw compressors and oil removal subsystems. This will reduce the weight of the He cryosystem by roughly $18,000 \mathrm{lbs}$. It also reduces the electrical supply and cooling requirements by approximately one MW each. Note, except for the rotating power connections, the He cryosystem components are all off-the-shelf commercial components.

- The magnet weights are for tunable magnets that can support a broad range of wavelengths of light. If only one wavelength of light is required by the mission, then permanent magnets plus small trim magnets can be used for the electron beam transport system. This option can significantly reduce the weight of the magnets.

- The wiggler weight would also be reduced if a single wavelength of light is used.

- The FEL produces minimal radiation when in operation and the radiation is negligible the instant the electron beam is turned off. A combination local shielding on selected components of the FEL and local shielding of the personnel areas minimizes the weight requirements.

- A large contingency has been included because a full engineering design has not yet been performed. However, the heaviest items, magnets and He refrigerator, are initial engineering estimates based on the compact FEL design shown and existing He refrigerators respectively.

\subsection{FEL Electrical System and Cooling}

Production of the one MW of laser optical power requires 3.0 MW of electrical power with a resultant requirement of 2.0 MW of cooling. The electrical power requirements would be higher except that direct rotary feeds from a jet engine are used to produce the compressed He, and high pressure takeoffs from the jet engine itself are also used to produce the cooling and liquid nitrogen needed in the He refrigerator cold box. Electric generation and cooling systems with these capacities are currently under development by the Air Force. The jet engine(s) for electric power generation and cooling require 1,000-1,500 lbs/hour of fuel depending upon the time spent at 40,000 feet and the power level of the FEL. This represents about seven percent of the fuel capacity for a Boeing 747 and will have minimal effects on the times between aerial refuellings for long duration missions. 
Table 1: FEL Subsystems and Weights

\begin{tabular}{|l|l|r|}
\hline \multicolumn{1}{|c|}{ Subsystem } & \multicolumn{1}{c|}{ Components } & Weight (lbs) \\
\hline Power generation \& air & Jet engines, rotating power, high pressure takeoffs & 7,000 \\
\hline Cooling & Heat removal, heat exchangers & 5,000 \\
\hline AC generation & Electric generator, power conditioning & 3,500 \\
\hline Electron beam gun & $500 \mathrm{kV}$ supply, gun, laser & 4,000 \\
\hline Cryostats & Injector, linac, beam stop & 8,500 \\
\hline RF & IOTs, waveguides, feedback & 2,000 \\
\hline Electron beam transport & Magnets, magnet chambers & 53,500 \\
\hline Wiggler & Magnetic elements, mirrors & 2,000 \\
\hline Vacuum & Piping \& pumps & 1,000 \\
\hline Mechanical support & Magnets, vacuum system & 5,000 \\
\hline Beam stop collector & Collector, shielding & 500 \\
\hline $2^{\circ} \mathrm{K}$ He & High pressure He compressors, expanders, heat & 48,000 \\
\hline Directional mirror & Mirror, optical transport & 20,000 \\
\hline Vibration damping & Active elements, supports & 1,000 \\
\hline Shielding & FEL area, personnel areas & 4,000 \\
\hline Contingency & & 15,000 \\
\hline Total & & 180,000 \\
\hline
\end{tabular}

Table 2 provides a listing of the subsystems consuming the 3.0 MW of electrical power. Table 3 provides a listing of the sources of the $2.0 \mathrm{MW}$ of waste heat to be removed by the cooling system. It is likely that high-pressure air from the jet engine compressors will be used for the cooling so as to minimize the large heat exchangers and weight associated with water based cooling systems.

Table 2: Electric Power Usage

\begin{tabular}{|l|r|}
\hline \multicolumn{1}{|c|}{ Subsystem } & $\mathrm{kW}$ \\
\hline DC injector: $0.5 \mathrm{~A}$ at $500 \mathrm{keV}$ & 300 \\
\hline Injector RF: $500 \mathrm{~kW} / 0.72$ & 700 \\
\hline Linac RF: $1,000 \mathrm{~kW} / 0.72$ & 1,400 \\
\hline Magnets & 400 \\
\hline Magnet power supplies & 50 \\
\hline He refrigerator cold box & 50 \\
\hline Misc (pumps, I\&C, etc.) & 100 \\
\hline Total & 3,000 \\
\hline
\end{tabular}

Table 3: Waste Heat Sources

\begin{tabular}{|l|r|}
\hline \multicolumn{1}{|c|}{ Subsystem } & \multicolumn{1}{c|}{$\mathrm{kW}$} \\
\hline DC injector & 50 \\
\hline Injector RF & 200 \\
\hline Linac RF & 400 \\
\hline Beam stop collector & 750 \\
\hline Magnets & 400 \\
\hline Magnet power supplies & 50 \\
\hline Misc & 150 \\
\hline Total & 2,000 \\
\hline
\end{tabular}




\subsection{FEL SRF Cavities and Cryomodule}

While the present Jefferson Lab FEL SRF system operates at 1,497 MHz, the optimal frequency for a MW class FEL is lower so that electron beam breakup effects are not an issue. A frequency near $750 \mathrm{MHz}$ is ideal. Jefferson Lab has just finished producing the cryomodules at $805 \mathrm{MHz}$ for the DOE Office of Science Oak Ridge National Laboratory's Spallation Neutron Source. Combining knowledge from this activity and recent R\&D in SRF including manufacturing, significant increases in cavity accelerating gradient and $Q$ have been achieved. Increases in accelerating gradient result in shorter cryomodules for the desired electron beam energy, and increases in $Q$ result in lower heat load on the cryogenic system.

Figure 4 shows an advanced design $750 \mathrm{MHz}$ five cell SRF cavity and how six cavities are joined together to make a 120 $\mathrm{MeV}$ cryomodule. ${ }^{13}$ Cavities can be added or removed to achieve the desired maximum electron beam energy for a given system. The electron beam breakup threshold for these cavities and cryomodules is significantly higher then required for MW and even multi MW FELs.

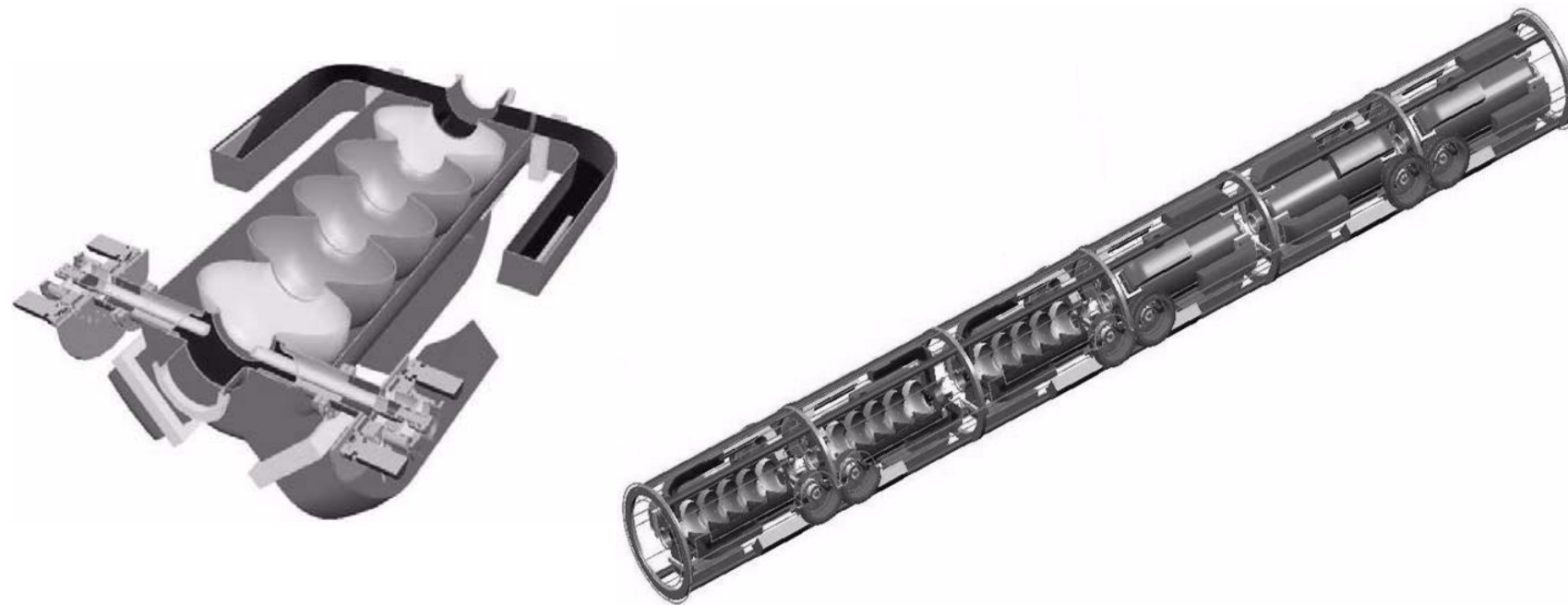

Figure 4: A five cell cavity with helium vessel, waveguide dampers and two Spallation Neutron Source style couplers that provides $20 \mathrm{MeV} /$ cavity $^{13}$ is shown on the left. In an example on the right, six cavities are placed in a single cryomodule for a resulting 120 $\mathrm{MeV}$ of acceleration. With room temperature end cans the total length of this cryomodule is $10.4 \mathrm{~m}$.

\subsection{FEL RF and RF Recovering Beam Stop}

For a one MW system, the injector requires $3.5 \mathrm{MW}$ of RF to accelerate the $0.5 \mathrm{amp}$ electron beam from $0.5 \mathrm{MeV}$ to 7.5 $\mathrm{MeV}$. The 1.0 MW of RF for the linac goes into optical beam. Using ERL concepts, the much higher RF powers present in the cavities in the linac come from the spent beam from the wiggler depositing all but $7.5 \mathrm{MeV}$ back into the cavities. When the spent electron beam leaves the linac on its way to the beam stop, it has 3.75 MW of power. By using an SRF cavity in the beam stop, $3.5 \mathrm{MW}$ of this RF power can be recovered and returned to the injector thereby reducing both the electric power and cooling requirements by 3.5 MW. To provide adequate stability in the control of the RF power, the injector is independently provisioned with $0.5 \mathrm{MW}$ of RF from an Inductive Output Tube (IOT). The RF recovering beam stop is shown in Figure 5.

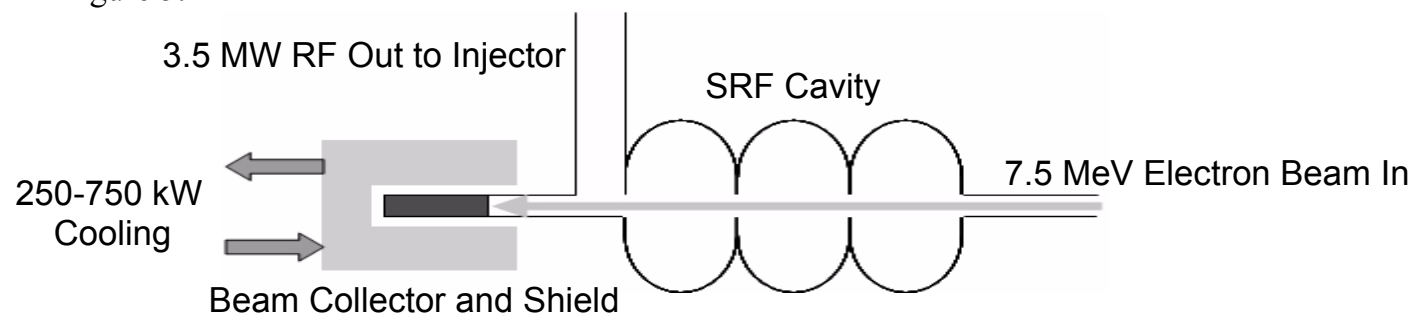

Figure 5: The RF recovering beam stop operates as a klystron. As RF builds in the cavity, $\sim 7 \mathrm{MeV}$ of the electron beam's energy is converted to RF for the injector. Depending upon how much of the injector's independent RF is required from the IOT for RF control, $250-750 \mathrm{~kW}$ of electron beam energy is deposited in the beam collector. 


\subsection{FEL Wiggler}

Depending on the range of wavelengths the FEL is operated at, the wiggler can be made of permanent magnetic materials. Figure 6 shows an example of designs currently under development. If a broad range wavelengths are desired, the wiggler's magnetic field may need to vary in conjunction with changes in the electron beam energy to produce the desired wavelengths of light at the MW level. Carefully wound superconducting helical coils can produce field strengths with similar characteristics as the permanent magnetic materials.

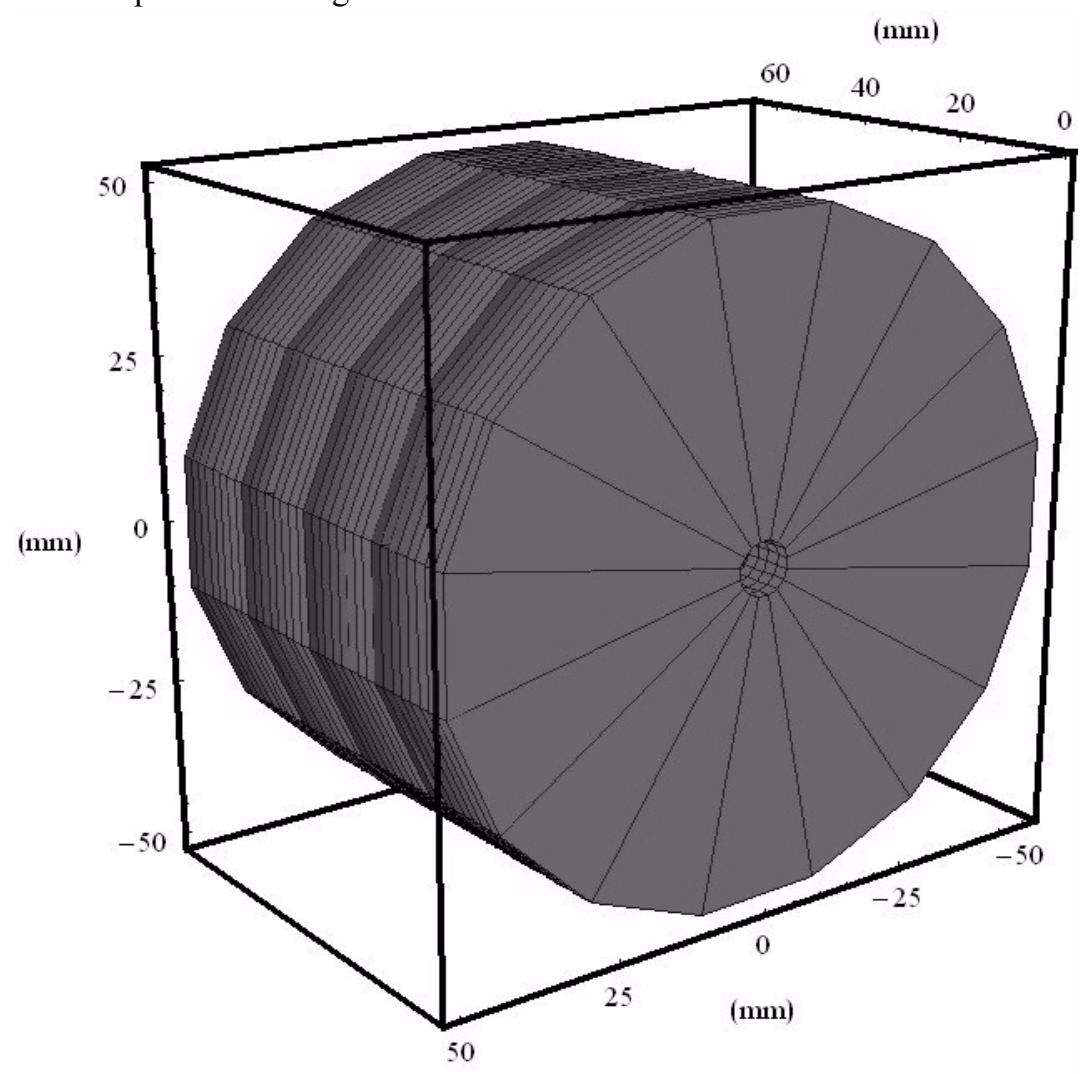

Figure 6: Four field rotations of a helical 1.3 Tesla neodymium-iron-boron permanent magnet wiggler are shown. ${ }^{14}$ With a 6 mm gap, $20 \mathrm{~mm}$ period, $10 \mathrm{rings} /$ period and 16 blocks/ring the field on axis is 0.94 Tesla with a resulting $\mathrm{k}=1.756$. A wiggler with these properties will produce $380(1,625) \mathrm{nm}$ light for an electron beam energy of $168(81) \mathrm{MeV}$. A helical wiggler has been selected to help minimize the production of harmonic light.

\subsection{FEL and Beam Director Optical Systems}

A key parameter in the FEL's gain is having the optical cavity's length (see Fig. 2) being tuned so that the subpicosecond pulses of light overlap with the subpicosecond pulses of the electron beam. This condition creates macro pulses of light with a time set by the round trip time for the light in the optical cavity. The FEL can operate with a single macro pulse or a string of them up to the condition where every available macro pulse has light. The power levels can vary from macro pulse to macro pulse by varying the pulse to pulse current produced off the photo cathode in the electron gun.

Another key set of parameters is having the electron beam's emittance being of very high quality so that the electron beam profile in the wiggler matches the optical Rayleigh length of the wiggler. When these and other conditions such as vibration control are met, the $\sim 10 \%$ of the light energy in the optical cavity that is extracted by the outcoupling optical mirror is of very high quality and the optical beam coming out of the beam director is to first order only diffraction limited. The beam director used for the Airborne Laser ${ }^{15}$, fully meets the primary mirror requirements for an airborne FEL. 


\subsection{FEL Light}

The FEL light can be steered and modulated for 4-D ( 3 space + time) tracking and targeting. In addition, a small fraction of the FEL's light can be lowered in wavelength and reinjected into the optical system with very high efficiency so that targets can be simultaneously tracked with multiple wavelengths. In addition the low power beams are very useful for running the adaptive optics needed to approach diffraction limited beam conditions.

Figure 7 shows an example of the FEL's light being varied in the time dimension. These same patterns can be produced in the secondary beams. The result is greatly enhanced target acquisition, tracking and targeting control.

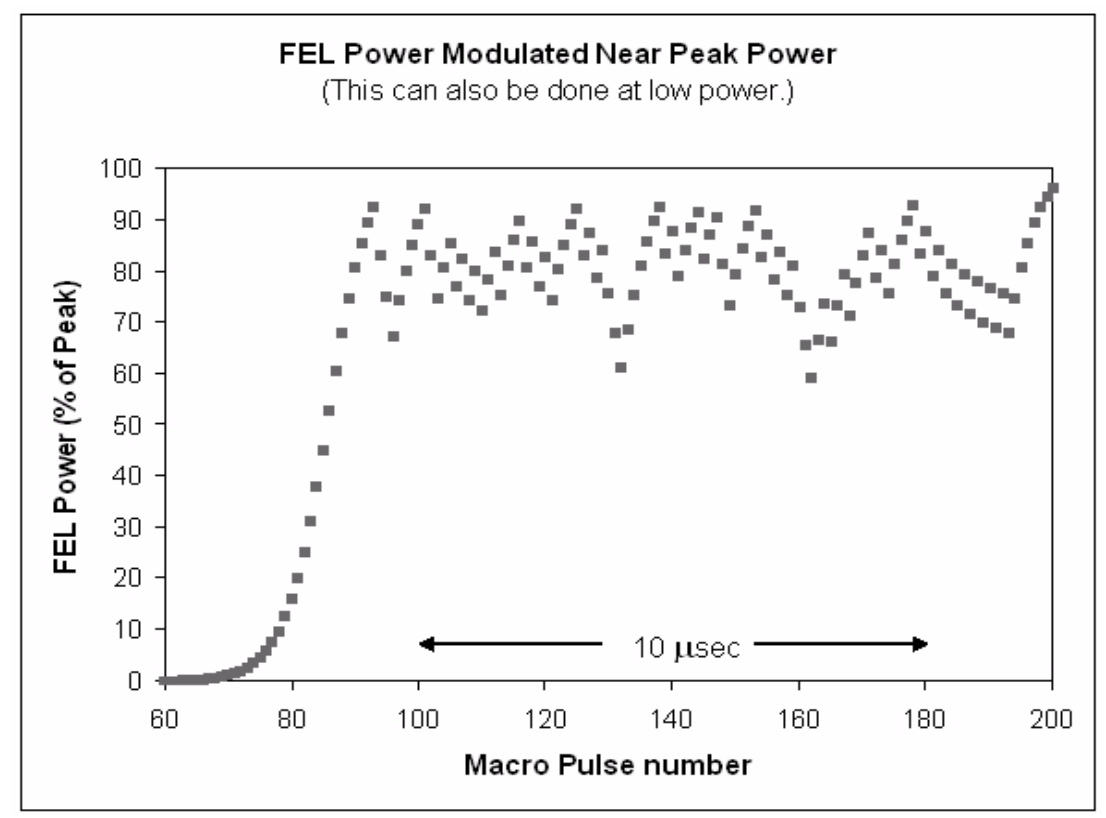

Figure 7: By pulse-to-pulse variation of the electron beam current, the FEL's light can be varied in the time dimension. This same pattern can then be observed in the reflected light from the target.

The wavelength of light selected for the FEL has multiple additional considerations such as using eye-safe wavelengths. Using selected wavelengths below $380 \mathrm{~nm}$ and above 1,400 $\mathrm{nm}$ can increase the Maximum Permissible Exposures (MPEs) by factors of more then twenty when compared to most wavelengths within that range. High MPEs are critical for certain missions. Shorter wavelengths decrease the diffraction effects for given size mirror. At the same time, the adaptive optics requirements for the shorter wavelengths are much more severe for dealing with air turbulence.

\section{MISSIONS}

A MW class laser on a large airplane such as a Boeing 747 is a strategic asset that can play a number of mission roles as has been discussed elsewhere, ${ }^{15,16,17}$ and the use of a FEL in this MW class role has been detailed in the prior sections of this paper. Equally important is that with the shrinking of MW class FEL in size and weight to fit on an airplane, additional mission areas become possible such as forward base protection and U.S. perimeter defense. All of these areas will integrate with high altitude optical relay mirror systems that are currently under development. ${ }^{16,18}$

\subsection{Forward Base Protection}

A compact 180,000 lb FEL can also be designed to be separated into 40,000 lb pieces, placed in truck trailers, loaded on airplanes, rapidly flown to any location, and then driven to the most desirable local location. The airplanes can be much smaller than a Boeing 747 thereby facilitating forward tactical deployments. Power generation could switch to diesel or other local sources. Within a few days after arrival at the final destination, the FEL can be assembled and made 
operational. If the time to operations is required to be exceptionally brief, the SRF cavities in their cryomodules can be transported at $4^{\circ} \mathrm{K}$ using currently available commercial liquid He air transport systems.

To achieve a several hundred mile effective range, the ground based FEL will be paired with a high altitude optical relay airship. With the FEL's unlimited magazine and multiple options for eye-safe wavelengths, this system will provide significant forward base protection and tactical operations capability. For many situations of interest, a similar capability can be achieved using a ship based FEL paired with a high altitude optical relay airship.

\subsection{U.S. Perimeter Missile Defense}

FELs are anticipated to achieve power levels beyond one MW within ten years, or sooner if pushed. This would make them capable of forming the basis of a U.S. perimeter missile defense. Being tunable, the FELs can be operated at eyesafe wavelengths making them deployable along the U.S. perimeter, even near population centers. As even higher power levels are desirable on targets, the perimeter defense concept is to spread the individual FELs along the U.S. perimeter such that any point of interest is in range of multiple FELs.

Key to the concept is minimizing the atmospheric affects of the perimeter based FELs by pairing them with a high altitude optical relay station airships as shown in Figure 8. This allows the FELs to point their beams near the vertical thereby minimizing the paths of the beams through the more dense portions of the atmosphere. The relay stations are mobile thereby allowing the light from the FELs to have minimal effects from clouds and turbulent air. In addition, using multiple FELs has the advantage of keeping the power density of the beams lower which will minimize if not eliminate the thermal blooming effects.

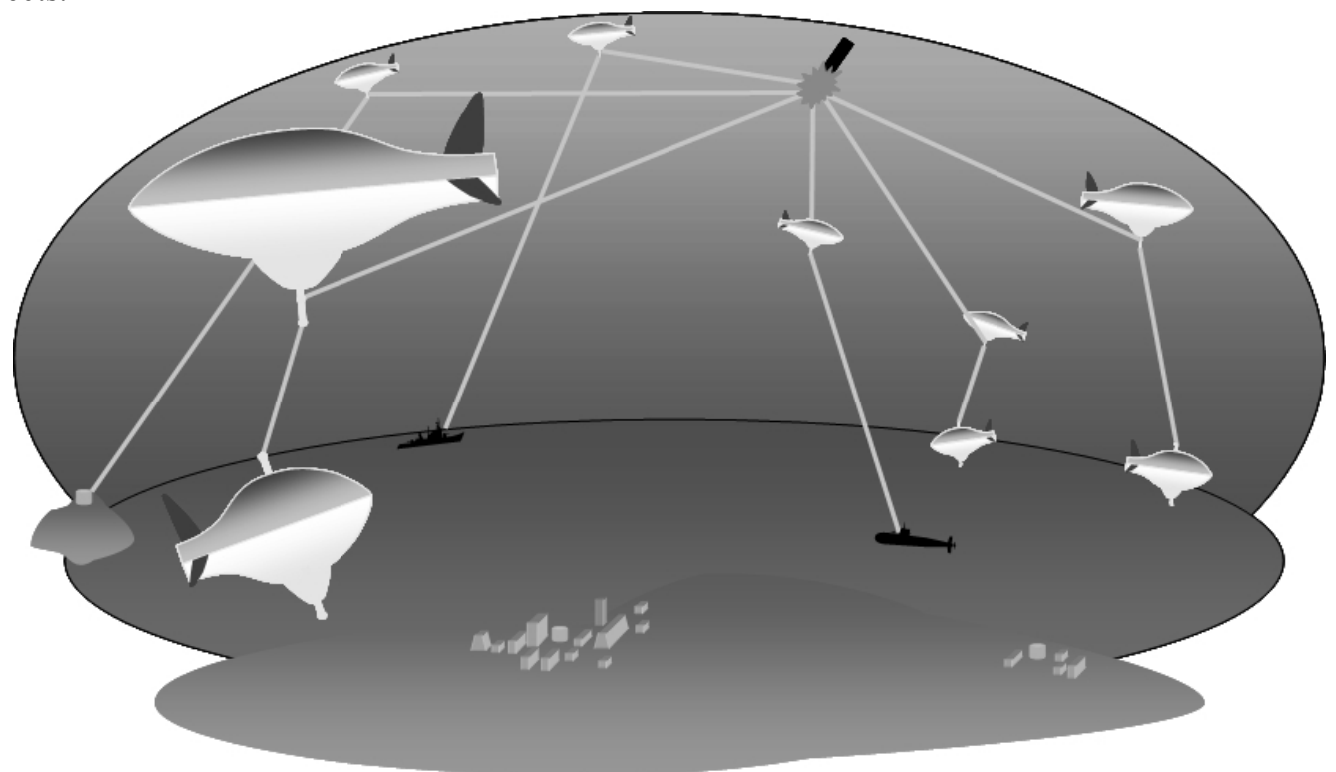

Figure 8: Using multiple FEL optical relay mirror pairs, the U.S. perimeter can be protected by a very robust system.

The perimeter based FELs can be ground, sea or airship based. Ground based FELs have the advantage of easy access to electrical power but can frequently be limited by weather. Sea based FELs can avoid some of the weather but will have to pass their beams through the maritime atmosphere. Airship based FELs are able to avoid almost all weather and regions of turbulent air along the optical paths to the relay stations. These FELs will be above most of the atmosphere where aerosols strongly influence the beam. Their fuel will easily last for weeks. With the reduction in atmospheric affects, the ranges and power densities on target substantially increase with the use of airship based FELs. Moving the FELs to the relay station altitude and eliminating the relay stations may be a possibility.

Following is a simple implementation example: Assume that 3.3 MW FELs are deployed at an average liner density of one every 100 miles along the U.S. perimeter. Assume that each FEL optical relay station pair has a range of 300 miles. 
The outcome is every place on the U.S. perimeter is within range of at least six of the FELs for a resultant 20 MW or more delivered on targets of interest.

Depending upon how the perimeters of Alaska, Hawaii and some of the U.S. territories are included, the U.S. perimeter can be considered to be up to 15,000 miles in length. In the implementation example, this results in the deployment of 150 FEL optical relay station pairs. If each pair averages $\$ 200 \mathrm{M}$, including the system integration cost, the total cost of the system is $\$ 30 \mathrm{~B}$.

At the level of 150 FEL optical relay station pairs, the complexity of the system can be well managed. Modest increases in the density of the pairs in appropriate areas would establish significant redundancy. Switching from standby to fully operational takes only a few seconds. Being FEL based, the system could run in operational mode for days on end. Targeting, of course, is at the speed of light.

\section{SUMMARY}

Compact, lightweight and highly efficient MW class FELs can be the key element for multiple missions. Airborne, airship and airborne transported versions can take on strategic missions at 40,000 feet in airplanes, more tactical missions for forward base protection, and the U.S. perimeter defense mission. Most of the required subsystems are already developed, commercially available or on the roadmaps for near-term development.

\section{ACKNOWLEDGEMENTS}

The authors gratefully appreciate the exchanges with the following people: FEL vision and plans - Fred Dylla (Jefferson Lab); system integration - Louis Marquet (DoD Ret.) and Alan Todd (Advanced Energy Systems); magnet systems George Biallas (Jefferson Lab); SRF cavities and beam stop RF recovery - Charles Reese (Jefferson Lab) and Robert Rimmer (Jefferson Lab); cryomodules - Warren Funk (Jefferson Lab); He cryogenic systems - Paul Brindza (Jefferson Lab), Claus Rode (Jefferson Lab), Daniel Gambet (Air Liquide) and David Grillot (Air Liquide); electric power generation - Jim Bray (General Electric), Donald Erbschloe (DOE), Charles Oberly (AFRL), Wayne Pearson (Honeywell), and Jay Vaidya (Electrodynamics Associates); wiggler - Duncan Scott (Daresbury); vibration - Mark Curtin (Boeing) and Robert Franzen (Lockheed Martin); eye safety - Patty Hunt, (Jefferson Lab); and mission integration - Jo Ann Erno (AF), Brian Jonasen (AF), John Haynes (AF), James Kotora, (MDA), James Mulroy (MDA), Denise Podolski (MDA), and Mark Stephen (AF).

This work was supported in part by the U.S. Department of Energy under contract DEAC05-84-ER40150, the Office of Naval Research, the Joint Technology Office, and the Commonwealth of Virginia.

\section{REFERENCES}

1. George R. Neil and Lia Merminga, "Technical Approaches for High Average Power FEL," Reviews of Modern Physics 74, 685 (2002).

2. S. V. Benson et al., "A $10 \mathrm{~kW}$ IRFEL Design For Jefferson Lab”, in Proceedings: 2001 Particle Accelerator Conference, Peter W. Lucas, Sara Webber, editors, IEEE, Piscataway, NJ (2001)

3. G. R. Neil et al., "Sustained Kilowatt Lasing in a Free-Electron Laser with Same-Cell Energy Recovery," Pays. Rev. Let. 84, 662 (2000).

4. S. Benson et al., "High Power Lasing in the IR Upgrade FEL at Jefferson Lab," presented at 2004 FEL Conference, Trieste, Italy, available at http://www.JACoW.org/

5. Jefferson Lab main website: http://www.jlab.org.

6. G. R. Neil et al., "Sustained Kilowatt Lasing in a Free-Electron Laser with Same-Cell Energy Recovery," Phys. Rev. Let. 84, 662 (2000).

7. L. Merminga, D. R. Douglas, and G. A. Krafft, "High-Current Energy-Recovering Linacs," Ann. Rev. Nucl. Part. Sci., 53 387-429 (2003). 
8. G.L. Carr, M.C. Martin, W.R. McKinney, K. Jordan, G.R. Neil and G.P. Williams "High Power Terahertz Radiation from Relativistic Electrons," Nature 420 153-156 (2002).

9. $\quad$ G.P. Williams, "High Power THz Synchrotron Sources”, Phil Trans. R. Soc. Lond. A362 403 (2004).

10. G. A. Krafft, "Compact high-power terahertz radiation source," Physical Review Special Topics - Accelerators and Beams, 7, 060704 (2004).

11. D. Douglas, JLAB TN-02-026, TN-03-032, and TN-03-036, see http://www.jlab.org/ douglas/ULTRAFEL/.

12. Boeing 747 website: http://www.boeing.com/commercial/747family/pf/pf_400f_prod.html (2004).

13. Courtesy R. Rimmer, Jefferson Lab (Private Communication).

14. Courtesy D. Scott, Daresbury (Private Communication).

15. Boeing ABL website: http://www.boeing.com/defense-space/military/abl/flash.html (2004).

16. D. L. Lamberson, E. Duff, D. Washburn, and C. Holmberg, "Whither High-Energy Lasers?" Air \& Space Power Journal, 18, Issue 1, April 1 (2004), available at http://www.airpower.maxwell.af.mil/airchronicles/apj/apj04/spr04/ lamberson.html.

17. T. Malik, "The Power of Light: An Airborne Laser for Missile Defense," available at http://www.space.com/ businesstechnology/airborne_laser_techwed_041117.html, (2004).

18. E. A. Duff and D. C. Washburn, "The Magic of Relay Mirrors," available at http://www.afrlhorizons.com/Briefs/ Oct04/DE0304.html (2004). 